\title{
EFFECT OF PILATES EXERCISES ON BLOOD GLUCOSE, URINARY PROBLEMS, SEXUAL SYMPTOMS, PAIN AND ANXIETY IN WOMEN WITH GESTATIONAL DIABETES MELLITUS
}

original paper

(1) University School of Physical Education in Wroclaw

DOI: https://doi.org/10.5114/hm.2021.103288

\section{GLENDA ROBERTA SILVA DO NASCIMENTO ${ }^{1}$, VANESSA DE SOUZA FERRAZ ${ }^{2}$, PATRICIA DE MEDEIROS VIEIRA WANDERLEY DE OLIVEIRA ${ }^{3}$, WILSON AYACH ${ }^{4,5}$, GUSTAVO CHRISTOFOLETTI ${ }^{6}$, ANA BEATRIZ GOMES DE SOUZA PEGORARE ${ }^{6}$}

\author{
${ }^{1}$ Student of the Physiotherapy course of the Integrated Institute of Health (INISA), Federal University of Mato Grosso \\ of South (UFMS), Campo Grande, MS, Brazil \\ ${ }^{2}$ Masters Student of the Graduate Program of Movement Sciences, Integrated Institute of Health (INISA), \\ Federal University of Mato Grosso of South (UFMS), Campo Grande, MS, Brazil \\ ${ }^{3}$ Maria Aparecida Pedrossian University Hospital, Brazilian Services Company, Federal University of Mato Grosso \\ of South (UFMS), Campo Grande, MS, Brasil \\ ${ }^{4}$ Maria Aparecida Pedrossian University Hospital, Federal University of Mato Grosso of South (UFMS), Campo Grande, \\ MS, Brazil \\ ${ }^{5}$ Faculty of Medicine, Federal University of Mato Grosso of South (UFMS), Campo Grande, MS, Brazil \\ ${ }^{6}$ Graduate Program of Movement Sciences, Integrated Institute of Health (INISA), Federal University of Mato Grosso \\ of South (UFMS), Campo Grande, MS, Brazil
}

\section{ABSTRACT}

Purpose. To verify the impact of Pilates training on blood glucose, urinary problems, sexual symptoms, pain and anxiety in women with gestational diabetes mellitus (GDM).

Methods. Overall, 25 pregnant women with GDM were randomly divided into an experimental and a control group. The participants allocated into the experimental group were submitted to 12 weeks of conventional treatment for GDM associated with supervised Pilates exercises. The control group were submitted to 12 weeks of conventional treatment, without the Pilates intervention. Fasting blood glucose was measured with a glucose monitor. Urinary symptoms were assessed by using the Short-Form International Consultation on Incontinence Questionnaire. For sexual function, the authors applied the Female Sexual Function Index. Maternal weight gain was obtained from the patients' medical records. Pain was assessed by visual analogue scale and localized by nordic questionnaire. Owing to a non-parametric pattern of the data, values were expressed as median and interquartile range. Inferential analysis was performed with the Mann-Whitney U test (to assess between-group comparisons) and Wilcoxon test (to assess pre- and post-intervention comparisons).

Results. Considering the baseline similarity of the groups regarding anthropometric and clinical variables, Pilates training improved fasting glucose, maternal weight gain, and urinary incontinence score as compared with the group submitted exclusively to conventional therapy $(p<0.05)$.

Conclusions. The results reinforce the beneficial effects of supervised Pilates training on reducing blood glucose, maternal weight gain, and urinary incontinence in pregnant women with GDM.

Key words: gestational diabetes, exercise movement techniques, Pilates, urinary incontinence, glycaemic control

\section{Introduction}

Gestational diabetes mellitus (GDM) is a common issue during pregnancy. The disease affects approximately $13.2 \%$ of pregnant women worldwide. The prev- alence increases with age at more than 45 years [1]. GDM can increase perinatal mortality and the risk of long-term comorbidities for mothers and children [2]. The condition is associated with higher rates of foetal macrosomia, stillbirth, neonatal metabolic disor-

Correspondence address: Ana Beatriz Gomes de Souza Pegorare, Physiotherapy Course, Institute of Health, Federal University of Mato Grosso do Sul, Avenida Universitária, s/n, University Sector, PO Box 549, e-mail: ana.pegorare@ufms.br

Received: March 6, 2020

Accepted for publication: July 6, 2020

Citation: Nascimento GRS, Ferraz VS, Oliveira PMVW, Ayach W, Christofoletti G, Pegorare ABGS. Effect of Pilates exercises on blood glucose, urinary problems, sexual symptoms, pain and anxiety in women with gestational diabetes mellitus. Hum Mov. 2021;22(4):36-45; doi: https://doi.org/10.5114/hm.2021.103288. 
ders, preeclampsia, and caesarean section [3]. About half of women with GDM will develop type 2 diabetes within 5 years after birth [4]. In the offspring, GDM is one of the main risk factors for developing obesity and glucose intolerance later in life [5].

Drug therapy and nutrition therapy are the cornerstones of the treatment of GDM in order to achieve glycaemic control and to reduce complications. In addition, a healthy lifestyle is recognized as an essential component of GDM management [6]. Lifestyle changes, self-monitoring of blood glucose, nutrition therapy, and physical activity are measures aimed at preventing hyperglycaemia and excessive weight gain during pregnancy [7].

Previous studies have shown that exercise increases the control of capillary blood glucose and can reduce or delay hypoglycaemic medication or insulin in women with GDM [4]. In this respect, insulin therapy is associated with an increase in hypertensive disorders and may increase the susceptibility to postpartum thromboembolic events [8].

Regarding exercise modalities, a recent systematic review concluded that cycling, walking, or circuit training performed 3 times per week for 40-60 minutes which resulted in an increase of $65-75 \%$ in the age-predicted basal heart rate was effective in improving glycaemic control in patients with GDM and in preventing the development of GDM in obese pregnant women $[9,10]$. These reviews recommended further studies to explore the impact of different combined exercise modalities on glucose metabolism in order to increase insulin sensitivity and to maximize their benefits in pregnancies with GDM $[9,10]$.

Pilates is an exercise modality based on 6 principles: involving the whole body, breathing, concentration, centralization, precision, and rhythm [11]. Pilates is a conscious approach in exercise, stimulating awareness of body structure, muscle recruitment, and body movement. Joseph Pilates claims that this set of corrective exercises promotes or controls voluntary or effective posture, stabilizing the posture muscles during dynamic movement and promoting mental vitality [12]. Pilates can be performed on a mat using the body's own weight as resistance or with Pilates equipment that provides resistance to stabilize and strengthen deep muscle groups [13].

Internationally, Pilates has become a form of exercise much sought by women to improve physical and mental health [14]. This modality advocates low-impact exercises with no sudden changes in direction, uses few repetitions, and works together with breathing and concentration to achieve some fluidity in movement execution [14].
Recent studies conclude that scientific evidence on the effects of Pilates exercises under special conditions such as pregnancy is still lacking, indicating the need for further research to determine the effectiveness of this method [15]. On the other hand, other studies found strong evidence to support the use of Pilates exercises to improve flexibility and dynamic balance [16] and functional capacity in sedentary women [17].

Specifically regarding GDM, the number of studies that evaluated the effects of Pilates exercises as an adjuvant treatment is scarce. The purpose of this study, thus, was to verify the impact of Pilates training on blood glucose, urinary problems, sexual symptoms, pain and anxiety in women with GDM.

\section{Material and methods}

\section{Participants}

A prospective clinical trial was conducted in which the participants were randomly divided into 2 independent groups: a Pilates group and a control group. The inclusion criteria involved age over 18 years, GDM diagnosis in accordance with the World Health Organization criteria, gestational age of 20-28 weeks, body mass index $\leq 45 \mathrm{~kg} / \mathrm{m}^{2}$, sedentary lifestyle (self-reported daily activities that did not increase the metabolic equivalent of task by 3 times the baseline), no smoking, clinical stability, and capability to participate in physical activities. Women who failed to attend the assessments or missed more than $25 \%$ of the training sessions were excluded.

\section{Therapeutic protocols}

The study involved 2 therapeutic protocols that were applied in women with GDM assisted at the University Hospital of the Federal University of Mato Grosso do Sul. In the Pilates group, the women underwent sessions of Pilates exercises combined with conventional treatment. The control group participants received only conventional treatment.

The selection of the participants was performed by convenience once only women who could attend the evaluation centre twice a week were included. Researchers were blinded to group assignment, and the division involved the use of envelopes.

A total of 25 participants were randomly divided into the Pilates ( $n=13$, mean age: $36.0 \pm 11.5$ years) and control ( $n=12$, mean age: $33.5 \pm 11.2$ years) groups (Figure 1). The method used to generate a random sequence, to allowing evaluate the possibilite to produce 
Excluded $(n=5)$

Unable to participate $(n=3)$

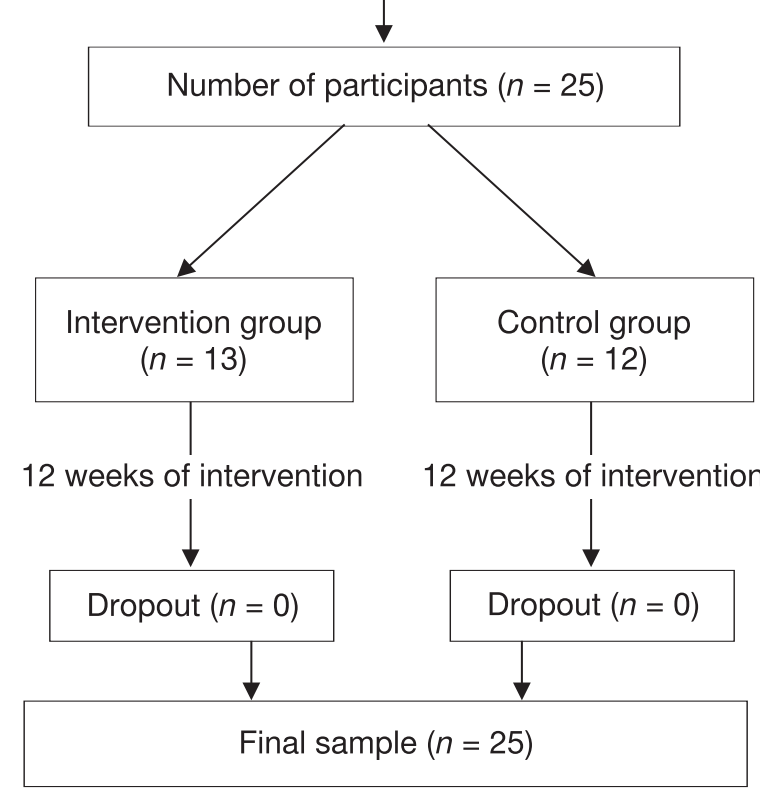

Figure 1. Study flow diagram

comparable groups, was by drawing the participants using sequential numbered, opaque and sealed envelopes.

\section{Pilates exercise intervention}

Women with GDM in the Pilates group received conventional treatment and were submitted to a structured Pilates exercise program supervised by a qualified physical therapist. The program consisted of 12 weeks of training, 2 sessions per week (55-60 minutes each), from 24-28 weeks of gestation to the end of the $3^{\text {rd }}$ trimester (36-40 weeks). A physical therapist specialized in women health was responsible for conducting the Pilates sessions.

The Pilates protocol used in the present study consisted of 10 different positions: spine stretch, spine twist, saw, mermaid, chest expansion, shoulder bridge, side kick - front and back, leg - small circles, scissors, and leg adduction. For progression of the exercises, the number of exercise repetitions was increased and the position of each exercise was changed from beginner to intermediate and advanced. The movements were repeated 6-8 times each. Figures 2-5 demonstrate some of the protocol exercises.

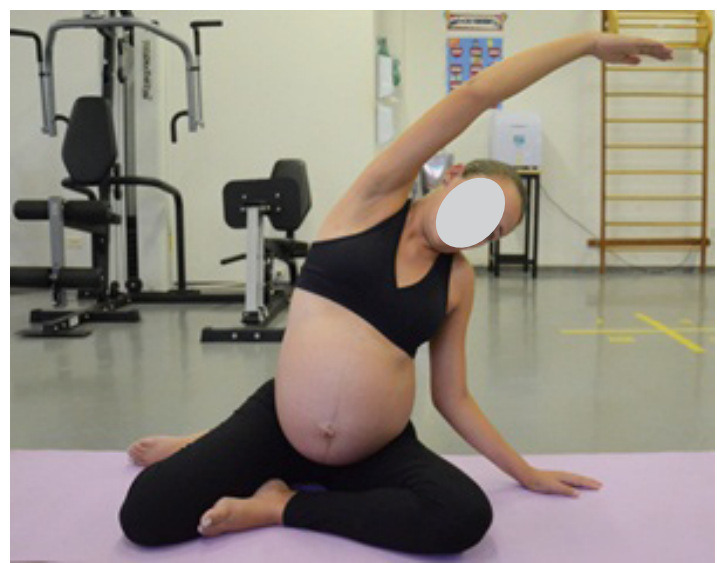

Figure 2. Mermaid

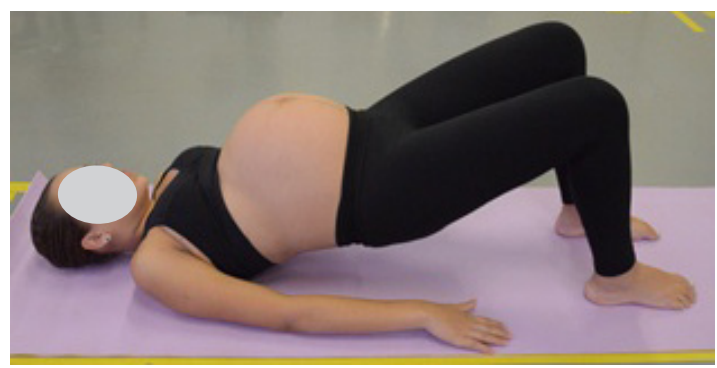

Figure 3. Shoulder bridge

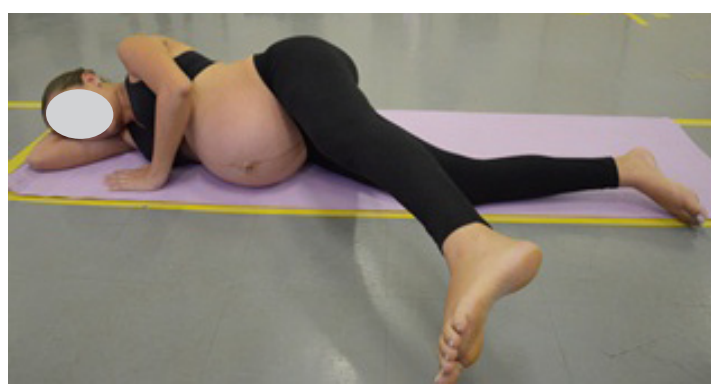

Figure 4. Side kick - front

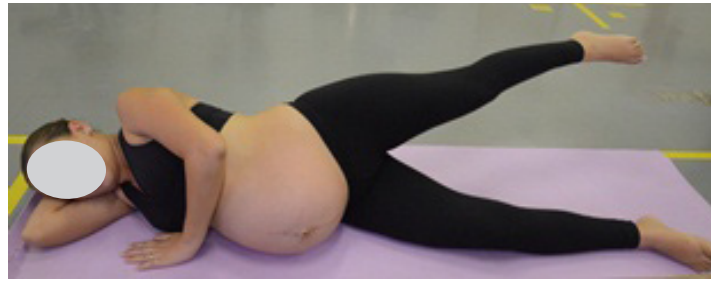

Figure 5. Side kick - back

\section{Conventional treatment}

The women receiving conventional treatment visited their obstetricians and nurses weekly (according to the hospital's protocol) until week 38-40 of gestation, when they delivered. According to the high-risk pregnancy manual of FEBRASGO [18], conventional treatment of GDM consists of a triad of diet, physical activity, and drug therapy (human insulin and, in specific cases, metformin). 
The diet prescribed for the participants consisted of $35 \mathrm{kcal} / \mathrm{kg} /$ day. The composition of the diet was as follows: $40-50 \%$ of carbohydrates, $15-25 \%$ of proteins, $25-30 \%$ of lipids, and less than $10 \%$ of unsaturated fats. The daily caloric intake was divided into 3 main meals (coffee, lunch, and dinner) and 3 snacks (morning, afternoon, and evening).

For standard physical activity, the pregnant women were instructed to walk on a flat surface for $30 \mathrm{~min}$ utes, 5 times a week, at a speed at which they could still talk. The medication of choice for treatment of diabetes mellitus was human insulin and, alternatively, metformin. A mean blood glucose concentration $\geq 120 \mathrm{mg} / \mathrm{dl}$ and/or $>30 \%$ of altered blood glucose measurements in the daily glycaemic profile were used as the criteria for the initiation of drug therapy and for dose adjustment.

The researchers remained in weekly contact with the participants to ensure that the activities were undertaken. The absence of undertaken activities for more than 2 weeks resulted in the exclusion of the subject from the study.

\section{Assessment instruments}

The Gynaecology and Obstetrics Outpatient Clinic of the university hospital includes the High Risk Pregnancy Treatment and Diagnosis Centre. All participants were first evaluated at the Clinic for the collection of anthropometric and clinical data (blood glucose, blood pressure, weight).

The International Consultation on Incontinence Questionnaire, Short Form (ICIQ-SF) was used to analyse the impact and severity of urinary incontinence [19]. The Hospital Anxiety and Depression Scale was applied to identify possible signs of mental health issues [20]. The Female Sexual Function Index (FSFI) served to analyse the patients' sexual function. FSFI is a self-administered questionnaire that evaluates 6 different domains of women's sexual function and activity: desire, sexual arousal, vaginal lubrication, orgasm, satisfaction, and pain [21]. The Nordic Musculoskeletal Questionnaire (NMQ) was used to locate and measure the report of musculoskeletal symptoms and, thus, facilitate the comparison of results between studies. The visual analogue scale allowed to measure pain intensity (from 0 to 10).

It is noteworthy that all instruments used were translated and validated to Portuguese and are suitable for the population studied.

\section{Sample size}

The sample size was calculated with the assumption of a type 1 error (alpha) of $5 \%$ and the statistical power (1-beta) of $80 \%$. With the design of a clinical trial with 2 independent groups and 2 assessment times, as well as an effect size of 0.6 , as previously reported by Lausen et al. [22], the minimum sample size should equal 20 participants, 10 in each group.

\section{Data analysis}

Data analyses involved descriptive and inferential statistics. For the descriptive statistics, the authors used median, interquartile range, and number of events. For inferential analysis, the categorical variables were compared with the use of the chi-square test. For the continuous variables, the Shapiro-Wilk test and Levene's test were first applied to verify if the requirements for the use of parametric tests were satisfied. Since most variables did not meet the requirements for parametric analyses, the authors applied the Mann-Whitney U test for assessments of between-group comparisons and the Wilcoxon test for assessments of the intragroup (pre-post treatment) analyses. For all analyses, significance was set at $5 \%$.

\section{Ethical approval}

The research related to human use has complied with all the relevant national regulations and institutional policies, has followed the tenets of the Declaration of Helsinki, and has been approved by the Human Research Ethics Committee of the Federal University of Mato Grosso do Sul (approval No.: 1.934.980) and registered in the Brazilian Registry of Clinical Trials (identifier: RBR-95xdzh).

\section{Informed consent}

Informed consent has been obtained from all individuals included in this study.

\section{Results}

Age, marital status, and pre-intervention weight of the intervention and control groups are shown in Table 1 . The participants were $35.0 \pm 11.0$ years old at the beginning of the study and both groups consisted exclusively of primigravidae. As shown in Table 1, the groups were similar in terms of all variables.

Table 2 shows body weight, fasting glucose, and postprandial glucose of women in the Pilates and control groups. As can be seen, the groups were homoge- 
Table 1. General characteristics of the participants

\begin{tabular}{|c|c|c|c|c|}
\hline \multicolumn{2}{|l|}{ Variable } & Pilates group & Control group & $p$ \\
\hline \multirow{2}{*}{\multicolumn{2}{|c|}{$\begin{array}{l}\text { Sample size }(n) \\
\text { Age (years) }\end{array}$}} & 13 & 12 & 0.841 \\
\hline & & $36.0(11.5)$ & 33.5 (11.2) & 0.567 \\
\hline \multirow{3}{*}{ Marital status (\%) } & Single & 92.3 & 58.3 & 0.063 \\
\hline & Married & 7.7 & 41.7 & \\
\hline & Divorced & 0 & 0 & \\
\hline \multicolumn{2}{|c|}{ Pre-intervention weight (kg) } & $85.0(20.0)$ & $90.0(26.7)$ & 0.087 \\
\hline
\end{tabular}

Chi-square test $p$-values for sample size and marital status

Mann-Whitney $U$ test $p$-values for age and pre-intervention weight

Table 2. Comparison of weight and blood glucose between groups

\begin{tabular}{|c|c|c|c|c|}
\hline Variable & Group & Before intervention & After intervention & $p$ (pre-post) \\
\hline \multirow{3}{*}{ Weight (kg) } & Pilates & $80.4(20.0)$ & $86.0(19.6)$ & $0.010 *$ \\
\hline & Control & $90.0(26.7)$ & $105.9(24.2)$ & $0.002^{*}$ \\
\hline & $p_{\text {(G1 vs. G2) }}$ & 0.080 & 0.003 & \\
\hline \multirow{3}{*}{ Fasting blood glucose (mg/dl) } & Pilates & $144.0(25.5)$ & $90.0(7.5)$ & $0.001^{*}$ \\
\hline & Control & $115.5(33.0)$ & $98.5(24.5)$ & 0.109 \\
\hline & $p_{\text {(G1 vs. G2) }}$ & 0.002 & 0.034 & \\
\hline \multirow{3}{*}{ Postprandial blood glucose (mg/dl) } & Pilates & $155.0(23.5)$ & $115.0(12.5)$ & $0.005^{*}$ \\
\hline & Control & $119.0(23.0)$ & $131.5(35.0)$ & 0.050 \\
\hline & $p_{\text {(G1 vs. G2) }}$ & 0.001 & 0.001 & \\
\hline
\end{tabular}

G1 - Pilates group, G2 - control group; ${ }^{*} p<0.05$

Mann-Whitney U test $p$-values for group comparisons (G1 vs. G2)

Wilcoxon test $p$-values for intragroup comparisons (pre-post)

Table 3. Level of anxiety and depression of the participants in the two groups

\begin{tabular}{lccccc}
\hline \multirow{2}{*}{ Variable } & \multicolumn{2}{c}{ Pilates group } & \multicolumn{2}{c}{ Control group } \\
\cline { 2 - 5 } & Yes & No & Yes & No & \\
\hline Pre-intervention anxiety (\%) & 38.5 & 61.5 & 41.7 & 68.3 & 0.870 \\
Post-intervention anxiety (\%) & 0.0 & 100.0 & 41.7 & 58.3 & $0.009 *$ \\
Pre-intervention depression (\%) & 53.8 & 46.2 & 50.0 & 50.0 & 0.848 \\
Post-intervention depression (\%) & 53.80 & 46.20 & 50.0 & 50.0 & 0.848 \\
\hline
\end{tabular}

* $p<0.05$

Chi-square test $p$-values

neous at baseline. Intragroup comparison showed a significant difference for weight $(p=0.002)$ in the control group. Pregnant women in the Pilates group benefitted from the physical therapy intervention in relation to fasting $(p=0.001)$ and postprandial glucose $(p=0.005)$.

Analysis of the level of anxiety and depression showed a significant improvement of anxiety in the Pilates group. The percentage of participants with symptoms of anxiety decreased from $38.5 \%$ to $0.0 \%$ in the experimental group $(p=0.009)$. Table 3 shows the anxiety level and depressive symptoms in both groups.
The application of the ICIQ-SF for the evaluation of urinary incontinence showed a significant improvement in the Pilates group over the 3 months of follow-up. The score of the participants decreased from $10.0 \pm 10.5$ to $2.0 \pm 5.5(p=0.005)$. No statistically significant difference in sexual function (FSFI) was observed between groups. Table 4 shows the pre- and post-intervention scores for urinary incontinence and sexual function in the Pilates and control groups.

The symptom of musculoskeletal pain was very common, reported by $76 \%$ of the participants. Among the musculoskeletal pain conditions, low back pain, 
Table 4. Urinary incontinence, sexual function, and pain scores in the Pilates and control groups

\begin{tabular}{llccc}
\hline Variable & Group & Before intervention & After intervention & $p$ (pre-post) \\
\hline \multirow{3}{*}{ Urinary incontinence } & Pilates & $10.0(10.5)$ & $2.0(5.5)$ & $0.005^{*}$ \\
& Control & $0.0(4.5)$ & $5.5(8.5)$ & 0.066 \\
& $p_{\text {(G1 vs. G2) }}$ & 0.017 & 0.414 & \\
\hline \multirow{3}{*}{ Sexual function } & Pilates & $18.2(17.4)$ & $19.8(9.9)$ & 0.477 \\
& Control & $19.5(20.3)$ & $16.8(18.6)$ & 0.109 \\
& $p_{\text {(G1 vs. G2) }}$ & 0.786 & 0.327 & \\
\hline \multirow{3}{*}{ Pain } & Pilates & $3.0(4.0)$ & $2.0(3.0)$ & $0.005^{*}$ \\
& Control & $4.0(3.0)$ & $5.0(1.0)$ & 0.007 \\
\hline
\end{tabular}

G1 - Pilates group, G2 - control group; ${ }^{*} p<0.05$

Mann-Whitney U test $p$-values for group comparisons (G1 vs. G2)

Wilcoxon test $p$-values for intragroup comparisons (pre-post)

with a prevalence of $60 \%$ (15 cases), was the most frequent in pregnant women. Table 4 shows the medians of the pain intensity scores assessed by the visual analogue scale. There was a reduction in pain intensity by the visual analogue scale in the Pilates group.

At baseline, $76.9 \%$ of women in the Pilates group were taking oral medication and $23.1 \%$ were administered injectable medication. In the control group, $66.7 \%$ of the women were using oral medication and $33.3 \%$ injectable medication. The chi-square test showed that the groups were similar on this pattern $(p=0.568)$. At the end of study, $23.1 \%$ of the participants of the experimental group were no longer taking medication and $76.9 \%$ used oral medication. In the control group, half of the women (50.0\%) used oral medication and the other half (50.0\%) injectable medication. The chi-square test indicated a significant difference between groups in this proportion $(p=0.007)$.

\section{Discussion}

The present results showed greater benefits in the intervention group (Pilates exercises combined with conventional treatment) compared with the control group (conventional treatment consisting of diet, medication, and walking).

Groups were homogeneous in relation to the clinical and anthropometric data (age, initial weight, and marital status). This similarity of the data permits to more clearly analyse the benefits caused by Pilates exercises. The results revealed a significant improvement in fasting and postprandial blood glucose levels, greater control of weight gain during pregnancy, improved urinary system function, and a reduction in the number of pain sites and anxiety in the Pilates group. In addition, the need for injectable medication was lower after the intervention in this group.

The control group exhibited a significant weight gain during pregnancy. In view of its various benefits, physical activity for pregnant women is recommended by the American Diabetes Association, American College of Obstetricians and Gynecologists, and Endocrine Society, as long as there is no contraindication concerning the woman's clinical or obstetric condition [23]. As part of GDM treatment, the recommendation is that pregnant women be encouraged to perform at least 30 minutes of moderate physical activity, at least 5 times a week, totalling 150 minutes per week, avoiding intervals of more than 2 consecutive days without exercise [18, 23].

Aerobic and resistance exercises are beneficial and both types should be performed to improve glycaemic control [24]. The experimental group was submitted to a combination of walking, which is an aerobic exercise, and Pilates, which involves resistance exercises. This combination had a greater impact on blood glucose levels, improving fasting glucose and postprandial glucose.

Previous studies have shown that physical exercise increases the rate of glucose uptake by the muscle cells during the exercise session and for a few hours after workout [25]. Exercise also improves the action of insulin, promoting glucose uptake into muscle and increasing the use of intracellular fatty acids, which, in turn, increases insulin sensitivity, stimulates glucose uptake, and decreases blood glucose levels for more than 20 hours within less than 70 hours [26]. Glucose uptake is also regulated by the duration and intensity of exercise performed. More intense exercises enhance the glucose lowering effect [25]. 
According to a systematic review on exercises among women with GDM, combined interventions with diet and exercises can have beneficial effects on blood glucose levels, especially postprandial blood glucose level [27]. A recent study showed that supervised exercises started early and maintained during pregnancy could reduce the risk of excessive maternal weight gain during pregnancy and the incidence of GDM [28]. Physical training during pregnancy has a positive effect on health and well-being of the motherbaby binomial, including appropriate maternal weight gain and a birth weight adequate for gestational age [29]. In fact, long-term studies have proved that women who exercise regularly are more likely to have a baby with a weight appropriate for gestational age, which decreases the risk of chronic diseases in adulthood, including cardiovascular diseases, obesity, and diabetes [30]. In the present study, Pilates training combined with walking, diet, and medication reduced maternal weight gain in the participants, possibly because of increased energy expenditure as a result of the 2 combined exercise modalities. In addition, the presence of the physical therapist accompanying the activities was a motivating factor for the correct execution of the exercises and for strictly following the prescribed diet.

Urinary incontinence, another common health problem during pregnancy, is caused by increased abdominal pressure, constipation, weight gain, increased intravesical pressure (greater demand for the continence mechanism). The application of ICIQ-SF showed incontinence in both groups at baseline; however, improvement of urinary symptoms was only observed in the Pilates group. This finding may be explained by the strengthening of the pelvic floor muscles. This muscle group is a component of the powerhouse, one principle of Pilates training [11, 12]. Another hypothesis is that worsening urinary incontinence in the control group was due to greater weight gain during pregnancy. This hypothesis is more plausible because, although the Pilates technique recommends pelvic floor muscle contraction, there is still no scientific evidence to recommend it as an exercise to treat urinary incontinence. Pelvic floor training is the most suitable type of exercise for the treatment of urinary incontinence [31].

Obesity and GDM have been increasing worldwide and may compromise female sexual function. In general, $56 \%$ of the 25 women enrolled in this study were at risk for sexual dysfunction symptoms (FSFI score $\leq 26)$. Some studies involving special populations such as women with GDM, pelvic organ prolapse, polycystic ovarian syndrome, and urinary incontinence have implied that weight gain negatively im- pacts some aspects of sexual function [32]. However, Pilates exercises did not improve sexual function in women with GDM.

Pregnancy is a major life event, which causes psychological, social, and hormonal changes. Significant changes in a short period of time may contribute to the development of mental disorders, such as anxiety and depression. A recent study found an association between diabetes and depressive symptoms [33]. A previous review demonstrated that $10-20 \%$ of women experienced some depressive symptoms during pregnancy. Among GDM women, the prevalence of depression during pregnancy ranged widely from $4.1 \%$ to $80.0 \%$ [34]. The susceptibility to depressive symptoms during pregnancy is possibly related to maternal cortisol levels. Pregnant women with diabetes appear to be at higher risk of developing depressive symptoms. In the present study, $52 \%(n=13)$ of the women had depressive symptoms and $40 \%(n=10)$ had anxiety symptoms at the initial assessment. No improvement of depressive symptoms was observed in either group. However, a decrease in the anxiety symptom score was established in the Pilates group.

A recent systematic review examined the effect of prenatal physical exercise on anxiety and depression during pregnancy and the postpartum period; the results showed no change in the odds of anxiety symptoms during or after pregnancy [35]. The study indicated that to achieve at least a moderate effect size in the impairment of the severity of prenatal depressive and anxiety symptoms, pregnant women needed to accumulate at least 644 MET-minutes/week of exercise (e.g., 150 minutes of moderate-intensity exercise, such as brisk walking, water aerobics, stationary cycling, or resistance training) [35]. It is possible that the increase of metabolic energy expenditure contributed to that finding (270 minutes of moderate-intensity exercise such as walking 5 times a week for $30 \mathrm{~min}$ utes and Pilates training twice a week for 60 minutes). In addition, the principles of Pilates training require concentration and breathing training, which consequently increase the feeling of well-being and relaxation-essential feelings during pregnancy [11, 12]. Another physical practice had similar effects on mental health and insulin levels among diabetic patients: yoga workouts. A recent systematic review of the literature has shown that prenatal yoga improves mental condition (stress, depression, and anxiety). It may also help reduce pelvic pain and improve the physical condition of pregnant women [36]. In another study, a metaanalysis revealed clinically improved effects of yoga intervention on glycaemic control and lipid profiles in 
pre-diabetic population. These results suggest that yoga intervention may be considered as an alternative approach to preventing type 2 diabetes mellitus [37].

Studies using the Pilates method in pregnant women are scarce and there is no previous research on Pilates training in women with GDM. However, Pilates exercise has been investigated in other populations, such as older adults and adults. A study compared home versus Pilates exercise programs for the rehabilitation of disability and found that supervised Pilates exercises had additional benefits, such as increasing adherence to exercise at home [38]. These results can be explained by the monitoring instructions and motivation provided by the exercise professional. The presence of the professional who accompanies the execution of all exercises, as well as of classmates, provides extra motivation for performing Pilates exercises [39]. We emphasize that interventions in homogeneous groups are a therapeutic option that favours the patients' adherence to treatment, especially among pregnant women with GDM since they have the same expectations of having a good delivery and healthy baby.

Pilates exercises can be used in combination with other treatments, are relatively inexpensive, and have few risks for the maternal-foetal binomial. These risks of hyperventilation, hypoglycaemia, and nausea are low, as the Pilates technique for pregnant women involves low-intensity training and low impact, and a professional monitors the breathing and fatigue of pregnant women during training [15].

\section{Conclusions}

Supervised Pilates exercises combined with conventional treatment were found to be beneficial for women with GDM, improving blood glucose levels (fasting and postprandial), urinary incontinence, and anxiety. In addition, Pilates exercises reduced maternal weight gain and the need to use injectable medication. In addition, Pilates exercises reduced maternal weight gain, the intensity of low back pain and the need to use injectable medication.

This study demonstrates the importance of professional monitoring of the exercise execution for a good outcome. More studies are needed to further investigate Pilates in women with and without GDM, seeking to make those exercises a routine among pregnant women.

\section{Acknowledgements}

Financial support was provided by the Federal University of Mato Grosso do Sul, Brazil, and by the Coor- dination for the Improvement of Higher Education Personnel (CAPES, finance code 001).

\section{Disclosure statement}

No author has any financial interest or received any financial benefit from this research.

\section{Conflict of interest}

The authors state no conflict of interest.

\section{References}

1. Melchior H, Kurch-Bek D, Mund M. The prevalence of gestational diabetes. Dtsch Arztebl Int. 2017;114(24): 412-418; doi: 10.3238/arztebl.2017.0412.

2. Bener A, Saleh NM, Al-Hamaq A. Prevalence of gestational diabetes and associated maternal and neonatal complications in a fast-developing community: global comparisons. Int J Womens Health. 2011;3:367-373; doi: 10.2147/IJWH.S26094.

3. Allehdan SS, Basha AS, Asali FF, Tayyem RF. Dietary and exercise interventions and glycemic control and maternal and newborn outcomes in women diagnosed with gestational diabetes: systematic review. Diabetes Metab Syndr. 2019;13(4):2775-2784; doi: 10.1016/j. dsx.2019.07.040.

4. Kim C, Newton KM, Knopp RH. Gestational diabetes and the incidence of type 2 diabetes: a systematic review. Diabetes Care. 2002;25(10):1862-1868; doi: 10.2337/diacare.25.10.1862.

5. Kawasaki M, Arata N, Miyazaki C, Mori R, Kikuchi T, Ogawa Y, et al. Obesity and abnormal glucose tolerance in offspring of diabetic mothers: a systematic review and meta-analysis. PLoS One. 2018;13(1): e0190676; doi: 10.1371/journal.pone.0190676.

6. Schmidt MI, Duncan BB, Reichelt AJ, Branchtein L, Matos MC, Costa e Forti A, et al. Gestational diabetes mellitus diagnosed with a 2-h 75-g oral glucose tolerance test and adverse pregnancy outcomes. Diabetes Care. 2001;24(7):1151-1155; doi: 10.2337/diacare.24. 7.1151.

7. Martis R, Crowther CA, Shepherd E, Alsweiler J, Downie MR, Brown J. Treatments for women with gestational diabetes mellitus: an overview of Cochrane systematic reviews. Cochrane Database Syst Rev. 2018;8(8): CD012327; doi: 10.1002/14651858.CD012327.pub2.

8. Cremona A, O’Gorman C, Cotter A, Saunders J, Donnelly A. Effect of exercise modality on markers of insulin sensitivity and blood glucose control in pregnancies complicated with gestational diabetes mellitus: a systematic review. Obes Sci Pract. 2018;4(5):455467; doi: 10.1002/osp4.283.

9. Wang C, Wei Y, Zhang X, Zhang Y, Xu Q, Sun Y, et al. A randomized clinical trial of exercise during pregnancy to prevent gestational diabetes mellitus and improve pregnancy outcome in overweight and obese preg- 
nant women. Am J Obstet Gynecol. 2017;216(4):340351; doi: 10.1016/j.ajog.2017.01.037.

10. Colberg SR, Sigal RJ, Fernhall B, Regensteiner JG, Blissmer BJ, Rubin RR, et al. Exercise and type 2 diabetes: the American College of Sports Medicine and the American Diabetes Association: joint position statement. Diabetes Care. 2010;33(12):e147-e167; doi: 10.2337/dc10-9990.

11. Pilates JH, Miller WJ. Return to life through contrology. New York: J. J. Augustin; 1945.

12. Pilates JH. Your health. Incline Village: Presentation Dynamics; 1934.

13. Latey P. The Pilates method: history and philosophy. J Bodyw Mov Ther. 2001;5(4):275-282; doi: 10.1054/ jbmt.2001.0237.

14. Lessen D. The PMA Pilates certification exam study guide. Miami: Pilates Method Alliance; 2014.

15. Mazzarino M, Kerr D, Wajswelner H, Morris ME. Pilates method for women's health: systematic review of randomized controlled trials. Arch Phys Med Rehabil. 2015;96(12):2231-2242; doi: 10.1016/j.apmr.2015.04. 005.

16. Cruz-Ferreira A, Fernandes J, Laranjo L, Bernardo LM, Silva A. A systematic review of the effects of Pilates method of exercise in healthy people. Arch Phys Med Rehabil. 2011;92(12):2071-2081; doi: 10.1016/j.apmr. 2011.06.018.

17. Rodrigues AQ, Martins FM, Barbosa AC, Figueiredo PS, Lima MO, Vieira ER. Effects of an eleven-week Pilates exercise program on progressive-speed walking capacity in sedentary young women: a pilot study. Hum Mov. 2016;17(2):102-106; doi: 10.1515/humo2016-0011.

18. FEBRASGO (Federação Brasileira das Associações de Ginecologia e Obstetrícia). Manual of high-risk pregnancy [in Portuguese]. Rio de Janeiro: FEBRASGO; 2011.

19. Tamanini JTN, Dambros M, D’Ancona CAL, Palma PCR, Netto NR Jr. Validation of the "International Consultation on Incontinence Questionnaire - Short Form” (ICIQ-SF) for Portuguese [in Portuguese]. Rev Saude Publica. 2004;38(3):438-444; doi: 10.1590/S003489102004000300015.

20. Pais-Ribeiro JL, Honrado A, Leal I. Contribution to the adaptation study of the Portuguese adaptation of the Lovibond and Lovibond depression anxiety stress scales (EADS) with 21 items [in Portuguese]. Psicol Saude Doencas. 2004;5(2):229-239.

21. Pacagnella RC, Martinez EZ, Vieira EM. Construct validity of a Portuguese version of the Female Sexual Function Index [in Portuguese]. Cad Saude Publica. 2009;25(11):2333-2344; doi: 10.1590/S0102-311X2 009001100004.

22. Lausen A, Marsland L, Head S, Jackson J, Lausen B. Modified Pilates as an adjunct to standard physiotherapy care for urinary incontinence: a mixed methods pilot for a randomised controlled trial. BMC Womens Health. 2018;18(1):16; doi: 10.1186/s12905-017-0503-y.

23. American College of Obstetricians and Gynecologists. ACOG Practice Bulletin No. 190: gestational diabetes mellitus. Obstet Gynecol. 2018;131(2):e49-e64; doi: 10.1097/AOG.0000000000002501.

24. Halse RE, Wallman KE, Newnham JP, Guelfi KJ. Home-based exercise training improves capillary glucose profile in women with gestational diabetes. Med Sci Sports Exerc. 2014;46(9):1702-1709; doi: 10.1249/ MSS.0000000000000302.

25. Halse RE, Wallman KE, Newnham JP, Guelfi KJ. Pregnant women exercise at higher intensity during 30 min of self-paced cycling compared with walking during late gestation: implications for $2 \mathrm{~h}$ postprandial glucose levels. Metabolism. 2013;62(6):801-807; doi: 10.1016/jmetabol.2012.12.009.

26. Turcotte LP, Fisher JS. Skeletal muscle insulin resistance: roles of fatty acid metabolism and exercise. Phys Ther. 2008;88(11):1279-1296; doi: 10.2522/ptj.2008 0018.

27. Harrison AL, Shields N, Taylor NF, Frawley HC. Exercise improves glycaemic control in women diagnosed with gestational diabetes mellitus: a systematic review. J Physiother. 2016;62(4):188-196; doi: 10.1016/j. jphys.2016.08.003.

28. Barakat R, Refoyo I, Coteron J, Franco E. Exercise during pregnancy has a preventive effect on excessive maternal weight gain and gestational diabetes. A randomized controlled trial. Braz J Phys Ther. 2019;23(2): 148-155; doi: 10.1016/j.bjpt.2018.11.005.

29. De Oliveira Santini C, Imakawa TDS, Duarte G, Quintana SM, Moisés ECD. Do the body mass index and the diagnosis of gestational diabetes mellitus influence the level of physical activity during pregnancy and postpartum? PLoS One. 2019;14(8):e0220947; doi: 10.1371/journal.pone.0220947.

30. Wang C, Zhu W, Wei Y, Feng H, Su R, Yang H. Exercise intervention during pregnancy can be used to manage weight gain and improve pregnancy outcomes in women with gestational diabetes mellitus. BMC Pregnancy Childbirth. 2015;15:255; doi: 10.1186/s12884-0150682-1.

31. Oliveira M, Ferreira M, Azevedo MJ, Firmino-Machado J, Santos PC. Pelvic floor muscle training protocol for stress urinary incontinence in women: a systematic review. Rev Assoc Med Bras. 2017;63(7):642-650; doi: 10.1590/1806-9282.63.07.642.

32. Bates JN, Pastuszak AW, Khera M. Effect of body weight on sexual function in men and women. Curr Sex Health Rep. 2019;11(1):52-59; doi: 10.1007/s11930019-00192-0.

33. Ponting C, Mahrer NE, Zelcer H, Dunkel Schetter C, Chavira DA. Psychological interventions for depression and anxiety in pregnant Latina and Black women in the United States: a systematic review. Clin Psychol Psychother. 2020;27(2):249-265; doi: 10.1002/cpp.2424. 
34. Ross GP, Falhammar H, Chen R, Barraclough H, Kleivenes $\mathrm{O}$, Gallen I. Relationship between depression and diabetes in pregnancy: a systematic review. World J Diabetes. 2016;7(19):554-571; doi: 10.4239/wjd.v7.i19.554.

35. Davenport MH, McCurdy AP, Mottola MF, Skow RJ, Meah VL, Poitras VJ, et al. Impact of prenatal exercise on both prenatal and postnatal anxiety and depressive symptoms: a systematic review and meta-analysis. Br J Sports Med. 2018;52(21):1376-1385; doi: 10.1136/ bjsports-2018-099697.

36. Kawanishi Y, Hanley SJB, Tabata K, Nakagi Y, Ito T, Yoshioka E, et al. Effects of prenatal yoga: a systematic review of randomized controlled trials [in Japanese]. Nihon Koshu Eisei Zasshi. 2015;62(5):221-231; doi: 10.11236/jph.62.5_221.

37. Ramamoorthi R, Gahreman D, Skinner T, Moss S. The effect of yoga practice on glycemic control and other health parameters in the prediabetic state: a systematic review and meta-analysis. PLoS One. 2019;14(10): e0221067; doi: 10.1371/journal.pone.0221067.

38. Natour J, de Araujo Cazotti L, Ribeiro LH, Baptista AS, Jones A. Pilates improves pain, function and quality of life in patients with chronic low back pain: a randomized controlled trial. Clin Rehabil. 2015;29(1):5968; doi: 10.1177/0269215514538981.

39. Tomas-Carus P, Garrido M, Branco JC, Castaño MY, Gómez MÁ, Biehl-Printes C. Non-supervised breathing exercise regimen in women with fibromyalgia: a quasiexperimental exploratory study. Complement Ther Clin Pract. 2019;35:170-176; doi: 10.1016/j.ctcp.2019.02.006. 